\title{
Accurate Modeling of Lossy Nonuniform Transmission Lines by Using Differential Quadrature Methods
}

\author{
Qinwei Xu, Student Member, IEEE, and Pinaki Mazumder, Fellow, IEEE
}

\begin{abstract}
This paper discusses an efficient numerical approximation technique, called the differential quadrature method (DQM), which has been adapted to model lossy uniform and nonuniform transmission lines. The DQM can quickly compute the derivative of a function at any point within its bounded domain by estimating a weighted linear sum of values of the function at a small set of points belonging to the domain. Using the DQM, the frequency-domain Telegrapher's partial differential equations for transmission lines can be discretized into a set of easily solvable algebraic equations. DQM reduces interconnects into multiport models whose port voltages and currents are related by rational formulas in the frequency domain. Although the rationalization process in DQM is comparable with the Padé approximation of asymptotic waveform evaluation (AWE) applied to transmission lines, the derivation mechanisms in these two disparate methods are significantly different. Unlike AWE, which employs a complex moment-matching process to obtain rational approximation, the DQM requires no approximation of transcendental functions, thereby avoiding the process of moment generation and moment matching. Due to global sampling of points in the DQM approximation, it requires far fewer grid points in order to build accurate discrete models than other numerical methods do. The DQM-based time-domain model can be readily integrated in a circuit simulator like SPICE. Unlike the commercial simulators, which cannot directly handle nonuniform transmission lines, the DQMs model nonuniform transmission lines by using the same procedure as model uniform lines at the same computational cost. Numerical experiments show that DQM-based modeling leads to high accuracy, as well as high efficiency. For both uniform and nonuniform multiconductor transmission lines, the proposed DQM technique is thrice faster than a commercial HSPICE simulator.
\end{abstract}

Index Terms-Differential quadrature method, interconnect modeling, nonuniform transmission line, transient simulation.

\section{INTRODUCTION}

D UE TO continuing very large scale integration (VLSI) feature size shrinking in CMOS and GaAs technologies, integrated circuits and systems such as multichip modules (MCMs) and system-on-a-chip (SOC) are becoming both larger in chip area and faster in operation. On-chip interconnects are experiencing increased sheet resistance and wiring inductance due to progressive interconnect scaling [1]. The interconnect scaling effect thus poses serious challenges to circuit simulators

Manuscript received November 21, 2000. This work was supported in part by a grant from the Multiuniversity Research Initiative and by a Dual-Use Program grant from the Office of Naval Research.

The authors are with the Department of Electrical Engineering and Computer Science, The University of Michigan at Ann Arbor, Ann Arbor, MI 48109 USA.

Digital Object Identifier 10.1109/TMTT.2002.803440. that employ simple $R C$ transmission modeling for interconnect delay estimation. As the length of on-chip global interconnects increases to a few centimeters or, as in ultrafast circuits, the signal rise/fall time becomes comparable to the time of flight across the wire, the inductance values of interconnects play a dominant role in determining signal waveforms and propagation delay. In order to estimate signal integrity and circuit speed, the delay modeling algorithms in circuit simulators must represent these interconnects as distributed $R L C$ transmission lines. In addition, the substrate effect and nonideal dielectric media require that the shunt conductance between the signal line and ground plane or bulk should be nonzero, and widths of interconnects are also shaped appropriately to match the impedances at corners and branching points. Therefore, at high speed and in large integrated systems like MCMs and SOCs, the metal interconnects should be treated as lossy and/or nonuniform transmission lines.

Transmission-line characteristics are usually represented by transcendental functions involving frequency and line length. As transmission lines are generally characterized in the frequency domain and are usually terminated with nonlinear loads, the time-domain models of transmission lines are needed so that the transient response can be calculated by applying convolution operation [2]. A considerable amount of research has been done in the literature to convert the frequency-domain solutions to time-domain responses. The first useful tool was the fast Fourier transform (FFT), which can be employed in most cases. When the inverse Fourier transform is directly used to find the time model, the computation complexity becomes proportional to the square of the simulation time, thus significantly slowing down the circuit simulators. Moreover, the FFT method has the intrinsic aliasing error problem.

Reduced-order macromodels have been popularly used. Asymptotic waveform evaluation (AWE) is the most well-known method to approximate general linear networks [3], [4]. In AWE, the Laplace-domain moments of the port characteristics are found to obtain a rational function via Padé approximation. The poles and residues of the rational function are used to describe the reduced-order macromodel. However, higher order moments lead to undesirable conditions when increasing the order of moments does not guarantee a better approximation. Furthermore, AWE may give a reduced macromodel, which includes unstable poles, although the original network is stable. In order to overcome these problems, another method, i.e., complex frequency hopping (CFH), has been developed as an extension to AWE [5], [6]. CFH performs 
the Taylor series expansion of the characteristic function at multipole points on the imaginary axis. Unlike traditional AWE, the frequency-hopping technique tries to preserve the poles rather than the moments. The residues of the poles are determined using a selected set of low-order moments generated at the various expansion points, which are referred to as hops. $\mathrm{CFH}$ preserves the poles of the transfer function rather than the moments, and it circumvents the ill conditions encountered in AWE. However, each hop of $\mathrm{CFH}$ requires its own expensive processing time and complex mathematical manipulations, while AWE only requires one. Two other methods, starting from the Telegrapher's equation and employing two-pole [7] or multipoint moment-matching techniques [8] can obtain reduced-order macromodels, which are extensions of $\mathrm{CFH}$.

On the other hand, direct applications of Padé approximation to the transmission-line problem have been performed [9], [10]. Using Padé approximation, the characteristic impedance and exponential propagation functions of the transmission line, which take the form of transcendental functions in the frequency domain, are converted into rational functions, so they can be analytically transformed into the time domain. This method gives the device models of transmission lines, just like the companion models of capacitance and inductance. However, the transient simulation of lossy transmission lines in the time domain requires computationally expensive convolution integrals, which become increasingly more expensive as the simulated time interval increases. In order to overcome this inefficiency, Lin and Kuh developed a recursive convolution with linear time efficiency using exponential functions [10]. Also, in [11], Chang and Kang developed a method called the piecewise recursive convolution, which is more efficient. The above methods extract the transmission-line delay separately, which are accurate for the time-delay evaluation. Another well-known technique, called the method of characteristics (MC) is efficient for solving Telegrapher's equations. It is well known that the MC can perfectly deal with lossless transmission line [12]. With generalized MC [12], a single transmission line can be modeled as a two-port network, which consists of two characteristic impedances and two voltage sources. Transmission-line models obtained by the above methods can be implemented into the existing circuit simulators such as SPICE. However, all these methods are based on the single-conductor transmission line having uniform width. It will be very complex to apply these methods to the coupled and/or nonuniform transmission lines.

Despite feasibility of dealing with a distributed transmission line, traditional circuit simulators can hardly handle practical VLSI systems. Besides functionality devices, large systems generally include a large number of state variables associated with the distributed interconnections and lumped $R L C$ elements that result from the modeling of the distribution network. Therefore, the direct use of a time-domain simulator like SPICE is prohibitively inefficient for realistic integrated systems and, thus, is limited only to the analysis of small systems. In order to overcome this difficulty, model reduction has been employed. A large system is first partitioned into nonlinear systems and linear systems and then the algorithms of model reduction are performed to linear parts. Krylov subspace techniques such as Lanczos and Arnoldi approaches are known for model reduc- tion. In Krylov-subspace-based methods, a set of orthonormal vectors is used to span the moment vector space and then the circuit matrix is projected into this vector space. Such a manipulation leads to multieigenvectors, while AWE converges only to the eigenvector corresponding to the eigenvalue with largest modulus. The use of orthonormal vectors helps obtain more than one eigenvalue, which is different from AWE. Both Padé via Lancoz (PVL) [13] and its multiport version matrix Padé via Lanczos (MPVL) [14] fall into this class of methods. A new direction for a passive reduced-order model, shown in [15], is based on congruence transformations, which analyzes the poles instead of matching the moments or eigenvalues. Nevertheless, an extended technique based on Arnoldi's method with congruence transformations is presented in the literature [16], in which the passive reduced-order interconnect macromodeling algorithm (PRIMA) was demonstrated as an effective approach to develop passive reduced-order models.

Although the algorithms of model reduction are well developed, it can only handle the finite systems in the forms of state variables. Specifically, the original system to be reduced should be described in the form of $A+s B=C$. Transmission lines, however, are represented by nonlinear partial differential equations (PDEs), which are infinite systems. Hence, it is inevitable to discretize the lines into discrete models in order to stamp them into the stencil of $A+s B=C$ prior to reduction process. As PDEs have been long approximately solved by finite-difference (FD) or finite-element (FE) methods [2], the discretization of transmission lines is far from a novel topic. A low-order finite method to model transmission lines gives a very understandable physical explanation of discretization schemes [17]. Such a popular approach for discrete modeling directly segments the line into sections, which is chosen to be a small fraction of the wavelength. From a mathematical viewpoint, this approach has low-order accuracy. Despite its simplicity, it has the disadvantage that the number of grid points depends on the minimum wavelength of interest, as well as the electrical length of the interconnect. Consequently, such an approach results in very large numbers of lumped elements for accurate modeling and, thus, sharply increases the number of state variables. A compact difference method is employed in the literature [18], which has fourth-order accuracy. Though this discretization is still a lower order finite method, the number of unknowns per wavelength required for highly accurate modeling is smaller and its dependence on the electrical length of the line is weaker.

The drawback of low-order finite methods can be removed by using the high-order finite methods or pseudospectral methods [19], [20]. The mathematical fundamental of FD schemes is the Taylor-series expansion. The scheme of a low-order finite method is determined by a low-order Taylor series, while the scheme of a high-order finite method is determined by a highorder Taylor series. In general, the high-order schemes have a high order of truncation error. Thus, to achieve the same order of accuracy, the mesh size used by the high-order schemes can be much less than that used by low-order schemes. As a result, the high-order schemes can obtain accurate numerical solutions using very few mesh points. Chebyshev polynomial representation has been used to model the transmission line in the literature [21], which serves as an example of high-order finite methods. 
In this paper, the differential quadrature method (DQM) is employed to model transmission lines. A numerical technique coming from the spectral method, the DQM was originally developed by mathematicians to approximately solve nonlinear PDEs [22]. As an alternative to FD and FE methods, the DQM gained use in solving differential equations in fluid mechanics, structural mechanics, and other engineering areas. The idea of the DQM is to quickly compute the derivative of a function at any point within its bounded domain by estimating a weighted linear sum of values of the function at a small set of points belonging to the domain. This paper applies the DQM to model interconnects in the following steps. At first, beginning from a Telegrapher's equation in the frequency domain, the DQM discretizes the differential equations as algebraic equations, which give the discrete model of the transmission line. Due to the super efficiency of the DQM, high accuracy can be obtained using moderate lower order approximation. Therefore, unlike other discrete models necessarily being reduced from high-order approximation to lower order one, the algebraic equations obtained by the DQM are then instead directly transformed into compact formation featuring rational approximations. The transmission line is thus modeled as a multiport device, and its time-domain model is obtained by applying inverse Laplace transform. By means of recursive convolution, the model takes the form of a companion model, whose computation complexity is linear with respect to the simulation time. Although the rational approximations in this method appear like the Padé approximations of AWE, it completely avoids the moment-matching process of AWE. There has been theoretical proof showing that the original DQM by Bellman is equivalent to the highest order FD method [23]. Due to this intrinsic feature of the DQM, the DQM-based modeling leads to higher computational efficiency than conventional finite methods do. Nevertheless, the afterwards ideas of developing the DQM offer much improvement in both applicability and accuracy of the DQM-based modeling of transmission lines.

The organization of this paper is as follows. In Section II, the mechanism of the DQM and several approaches to determine differential quadrature (DQ) coefficients are outlined. The discretization of Telegrapher's equations and compact modeling are discussed in Section III. Section IV gives a heuristic rule for determining the DQM's order and accuracy. In Section V, several numerical examples are presented and the results of various DQM algorithms have been compared with the results obtained using HSPICE.

\section{DQM}

Classical numerical techniques such as FD and FE methods have been fully developed in the literature and are widely used to numerically solve differential equations. Despite their wide popularity and uses, they require computationally prohibitive time to solve large problems. Consequently, such direct numerical methods have been rather sparingly used in modeling multiconductor lossy transmission lines. Although the DQM is a numerical approximation, it can quickly calculate the derivative of a function by sampling the exact values of the function at a small set of grid points. Therefore, it involves far fewer computing quantities than the FD and FE methods, nevertheless retaining the simple features of the direct numerical techniques.

\section{A. Approximation of Derivative in Terms of Function Values}

The integral quadrature method is used to approximate the definite integral of the following form:

$$
\int_{0}^{1} u(x) d x=\sum_{i=1}^{N} a_{i} u\left(x_{i}\right)
$$

where $x \in[0,1]$ and $0=x_{1}<x_{2}<\cdots<x_{N}=1$, $i=1,2, \ldots, N$. Equation (1) is called the $N$ th-order integral quadrature application. Following the concept of integral quadrature, the DQM can be employed to approximate the derivative of the distributed voltages and currents along transmission lines [24] given by

$$
\frac{\partial}{\partial x} u\left(x_{i}, t\right)=\sum_{j=1}^{N} a_{i j} u_{j}(t)
$$

for first-order derivative and

$$
\frac{\partial^{n}}{\partial x^{n}} u\left(x_{i}, t\right)=\sum_{j=1}^{N} a_{i j}^{(n)} u_{j}(t)
$$

for higher order derivative, where $x \in[0,1], 0=x_{1}<x_{2}<$ $\cdots<x_{N}=1$, and $i, j=1,2, \ldots, N . u(x, t)$ represent the distributed voltage $v(x, t)$ or current $i(x, t)$, and $u_{j}(t)=$ $u\left(x_{j}, t\right)$.

As the application in this paper only concerns the first-order derivatives of distributed voltages and currents along transmission lines, we need only to study (2), which is called the $N$ th-order DQ approximation.

In both the integral quadrature and DQ, the quadrature coefficient $a_{i}$ or $a_{i j}$ can be determined by using interpolating polynomials. Integral quadratures with a variety of interpolating polynomials are fully developed in the literature, while the DQs are still in the developmental stage. The DQM has obvious applications in the numerical solution of ordinary and PDEs with known boundary conditions. In general, the interpolation formulas are expressed in two ways, i.e., either in terms of differences of the function or in terms of values of the function. The derivatives of a function can also be expressed in the same manner in both cases. The first case, i.e., the approximation of derivatives with differences, provides the basis of many methods for solving differential equations. Well-known methods such as Euler, Gear, etc. are examples of the low-order FD and FE methods. Although these methods lead to high accuracy given enough grid points, they suffer from large computation time and their stable conditions are extremely strict. On the contrary, the second case, i.e., the approximation of derivatives with function values, gives moderately accurate results at a lower cost by using fewer grid points; thus, taking less computation time and having easy stable conditions. 
The key procedure to this technique is to determine the weighting coefficients $a_{i j}$. Following the concept of the weighting residual method, a suggested way by Bellman is to let (2) be exact for test functions

$$
g(x)=\left\{1, x, x^{2}, \ldots, x^{N-1}\right\}
$$

By substituting every item in the function set into (2), the following Vandermonde matrix equations are obtained:

$$
\left[\begin{array}{ccc}
1 & \cdots & 1 \\
x_{1} & \cdots & x_{N} \\
x_{1}^{2} & \cdots & x_{N}^{2} \\
\vdots & \ddots & \vdots \\
x_{1}^{N-1} & \cdots & x_{N}^{N-1}
\end{array}\right]\left[\begin{array}{c}
a_{i 1} \\
a_{i 2} \\
a_{i 3} \\
\vdots \\
a_{i N}
\end{array}\right]=\left[\begin{array}{c}
0 \\
1 \\
2 x_{i} \\
\vdots \\
(N-1) x_{i}^{N-2}
\end{array}\right]
$$

for $i=1,2, \ldots, N$. Due to the property of the Vandermonde matrix equation, the coefficient $a_{i 1}, a_{i 2}, \ldots, a_{i N}$ are uniquely determined by solving the above equations.

Since the accuracy of the DQM depends on the estimation of $a_{i j}$ coefficients, in (2), an error term $e_{i}=e\left(x_{i}, t\right)$ in the $N$ th-order DQ is added to approximate a first-order derivative as follows:

$$
\frac{\partial}{\partial x} u\left(x_{i}, t\right)=\sum_{j=1}^{N} a_{i j} u_{j}(t)+e_{i}
$$

It has been established in the literature [22] that if all the following conditions are satisfied:

- the first, second, ..., and $N$ th derivative $u^{(1)}(x, t)$, $u^{(2)}(x, t), \ldots, u^{(N)}(x, t)$ are continuous;

- $u^{(N)}(x, t) \leq K$

then the error can be evaluated by

$$
e_{i} \leq K \frac{h^{N-1}}{(N-1) !}
$$

where $h=\max \left(x_{j+1}-x_{j}\right), j=1,2, \ldots, N-1$. The above equation shows that the DQM approximation has an accuracy of $N$ th order.

\section{B. Determination of $D Q$ Coefficients}

Though the DQ coefficients can be obtained by solving (5), it has been theoretically proven that the method using power functions cannot give higher accuracy when the order of the DQM is too large. When $N$ is large $(N>10)$, the matrix is ill conditioned and its inversion is numerically difficult [22]. As a developed version of the original DQM, the generalized differential quadrature (GDQ) [25] and generalized collocation method [26] are presented. In the practical application of a distributed transmission line, the voltage or current $u=u(x, t)=$ $\{v(x, t), i(x, t)\}$ can be approximated by means of interpolation as follows:

$$
u(x, t) \simeq u^{N}(x, t)=\sum_{i=1}^{N} L_{i}(x) u_{i}(t), \quad x \in[0,1]
$$

where $u_{i}(t)=u\left(x_{i}, t\right)$ and $L_{i}(x)$ is an interpolation function determined by $x_{i}$ and interpolating formulation. As a result, the coefficients in (2) can be determined by

$$
a_{i j}=\frac{d}{d x} L_{i}\left(x_{j}\right)
$$

1) Polynomial Differential Quadrature (PDQ): If $L_{i}(x)$ in (6) is given as Lagrange polynomials by the following expression:

$$
L_{i}(x)=\frac{\prod_{k \neq i}\left(x-x_{k}\right)}{\prod_{j \neq i}\left(x_{i}-x_{j}\right)}
$$

then calculations from (7) provide the following result:

$$
\begin{aligned}
a_{i j} & =\frac{1}{\left(x_{i}-x_{j}\right)} \frac{\prod_{k \neq j}\left(x_{j}-x_{k}\right)}{\prod_{k \neq i}\left(x_{i}-x_{k}\right)}, \quad i \neq j \\
a_{i i} & =\sum_{i \neq j} \frac{1}{x_{i}-x_{j}} .
\end{aligned}
$$

This process shows that the DQ method is closely related to the collocation or pseudospectral method [19]. Its principal advantages over the latter, however, lies in its simplicity of using grid spacing without any restriction. The grid points in the original DQ method are supposed to be equispaced collocations, while those of GDQ methods can be chosen arbitrarily.

If the grid points are the zeros of an $n$ th-order Chebyshev polynomial, the following very simple formulas can be obtained for computing the DQ weighting coefficients:

$$
\begin{aligned}
& a_{i j}=\frac{(-1)^{i+j}\left(r_{N}-r_{1}\right)}{r_{i}-r_{j}} \sqrt{\frac{1-r_{j}^{2}}{1-r_{i}^{2}}}, \quad i \neq j \\
& a_{i i}=\frac{\left(r_{N}-r_{1}\right) r_{i}}{2\left(1-r_{i}^{2}\right)}
\end{aligned}
$$

where $r_{1}, r_{i}, r_{j}$, and $r_{N}$ are, respectively, first, $i$ th, $j$ th, and $N$ th zeros of an $N$ th-order Chebyshev polynomial. This result is agreeable with that derived by using the presentation of Chebyshev polynomials in [21]. No matter if the test functions are power series, Lagrange polynomials, or Chebyshev polynomials, it is easy to show that the high-degree polynomial in every case constitutes an $N$-dimensional linear vector space $V_{N}$ with respect to the operation of addition and multiplication. According to the properties of a linear vector space of polynomials, function set (4) spans an $N$-dimension subspace of a complete orthogonal base in this linear space, therefore, any other $N$ th-order polynomial in $V_{N}$ can be uniquely expressed as a linear combination of (4). Furthermore, if all the base polynomials of (4) satisfies a linear relationship [see (2)] so does any $N$ th-order polynomial in the linear space. In the $N$-dimension linear vector subspace, there may exist several $N$-dimension subsets of orthogonal base polynomials. Each subset of base polynomials can be uniquely expressed by another subset, and each different subset lead to a set of unique DQ coefficients. 
TABLE I

MeAn SQuAred ERror: DQM Versus FD ( $N$ : THE Number OF GrID POINTS)

\begin{tabular}{l||c|c|c|c|c|c|c|c}
\hline$N$ & 5 & 10 & 15 & 20 & 25 & 50 & 75 & 100 \\
\hline FD & 0.0181 & 0.0043 & 0.0019 & 0.0010 & $6.6571 \mathrm{e}-4$ & $1.6430 \mathrm{e}-4$ & $2.2705 \mathrm{e}-5$ & $4.0807 \mathrm{e}-5$ \\
\hline \hline$N$ & 5 & 7 & 9 & 11 & 13 & 15 & 17 & 19 \\
\hline PDQ & $4.3367 \mathrm{e}-5$ & $7.5297 \mathrm{e}-9$ & $4.6001 \mathrm{e}-13$ & $1.3445 \mathrm{e}-13$ & $1.4850 \mathrm{e}-13$ & $1.4085 \mathrm{e}-13$ & $1.2589 \mathrm{e}-13$ & $1.9603 \mathrm{e}-13$ \\
\hline HDQ & 0.0101 & $6.9302 \mathrm{e}-4$ & $5.2277 \mathrm{e}-5$ & $4.1760 \mathrm{e}-6$ & $3.4524 \mathrm{e}-7$ & $2.9192 \mathrm{e}-8$ & $2.5292 \mathrm{e}-9$ & $2.1988 \mathrm{e}-10$ \\
\hline
\end{tabular}

In this sense, the approaches shown as (5), (9), and (10) can be called the PDQ.

2) Harmonic Differential Quadrature $(H D Q)$ : For electronic circuits and systems, the Fourier series may be a better approximation of the true solution than polynomial expansion. An $N$ th-order Fourier expansion is a linear combination in an $N$-dimension linear subspace, which is spanned by the following orthogonal base:

$$
\begin{array}{r}
g(x)=\{1, \sin \pi x, \cos \pi x, \sin 2 \pi x, \cos 2 \pi x, \ldots, \\
\left.\sin \frac{N-1}{2} \pi x, \cos \frac{N-1}{2} \pi x\right\}
\end{array}
$$

where $N$ is the number of grid points that is normally an odd number. In order to determine the weighting coefficients using sine and cosine functions, let (2) be exact when test functions take the set in (11), then the weighting coefficients are determined by

$$
\sum_{j=1}^{N} a_{i j} g\left(x_{j}\right)=\frac{d}{d x} g\left(x_{i}\right), \quad i=1,2, \ldots, N
$$

Further study shows that $u(x, t)$ in this case is approximated by interpolation

$$
u(x, t) \simeq u^{N}(x, t)=\sum_{i=1}^{N} F_{i}(x) u_{i}(t), \quad x \in[0,1]
$$

where

$$
F_{i}(x)=\frac{\prod_{k \neq i} \sin \left(\left(x-x_{k}\right) \pi / 2\right)}{\prod_{k \neq i} \sin \left(\left(x_{i}-x_{k}\right) \pi / 2\right)}
$$

and the coefficients of the HDQ can be calculated by

$$
a_{i j}=\frac{d}{d x} F_{i}\left(x_{j}\right)
$$

whose explicit computational formulas are

$$
\begin{aligned}
& a_{i j}=\frac{\pi / 2}{\sin \left(\left(x_{i}-x_{j}\right) \pi / 2\right)} \frac{\prod_{k \neq j} \sin \left(\left(x_{j}-x_{k}\right) \pi / 2\right)}{\prod_{k \neq i} \sin \left(\left(x_{i}-x_{k}\right) \pi / 2\right)}, \quad i \neq j \\
& a_{i i}=-\sum_{k \neq i} a_{i k} .
\end{aligned}
$$

\section{Properties of $D Q$ Operators}

Once the positions of selected points are fixed, each of the aforementioned approaches gives constant DQ coefficients, no matter in what applications the differential equations appear. In general, the set of points are selected carefully so that they are symmetric with respect to the center of the domain; alternatively, they can be equally spacing points over the domain. At each point $x=x_{i}$, the derivative can be calculated as

$$
\frac{\partial}{\partial x} u_{i}(t)=\left[a_{i 1} \cdots a_{i N}\right]\left[u_{1}(t) \cdots u_{N}(t)\right]^{T}
$$

where $u_{i}(t)=u\left(x_{i}, t\right)$. Therefore, all the $N$ th-order DQ coefficients constitute a matrix $\mathbf{A}$, which is called the $N$ th-order DQ coefficient matrix. Hence, a continuous derivative in the domain $[0,1]$ is discretized to a linear operator

$$
\frac{\partial}{\partial x} \mathbf{u}=\mathbf{A} \mathbf{u}
$$

where $\mathbf{u}=\left[u_{1}(t) \cdots u_{N}(t)\right]^{T}$. For instance, the equally spacing fifth-order DQ coefficient matrix determined by (4) is as follows:

$$
\mathbf{A}=\left[\begin{array}{ccccc}
-25 / 3 & 16 & -12 & 16 / 3 & -1 \\
-1 & -10 / 3 & 6 & -2 & 1 / 3 \\
1 / 3 & -8 / 3 & 0 & 8 / 3 & -1 / 3 \\
-1 / 3 & 2 & -6 & 10 / 3 & 1 \\
1 & -16 / 3 & 12 & -16 & 25 / 3
\end{array}\right]
$$

The above matrix has the property of inverse symmetry with respect to the central point. In fact, every DQ coefficient matrix has such a property, which reduces the computation time for finding the coefficients by $50 \%$. The following lemmas formally describe the properties of DQM operators:

Lemma 1: A DQM operator is inverse symmetric with respect to the central point of the matrix.

Lemma 2: An $N$ th-order DQM operator has a rank of $(N-$ 1).

Lemma 3: The sum of a row of a DQM operator matrix is zero.

It is observed that the DQ method is an extension of the well-known difference method, which can also lead to a matrix $\mathbf{A}$ (FD operator). However, the sparse difference operator consists of tridiagonal matrices, while the coefficient matrix of the DQ method is not sparse. The difference method uses neighboring points to numerically compute the derivative at a point, while the DQ method employs the mesh points in the whole domain. In that sense, the former can be considered as local approximation, while the latter leads to the global approximation. Consequently, DQ coefficients are global coefficients and they yield highly accurate numerical solutions by using much fewer 
grid points. Table I shows the mean squared error for a signal at time $t, v(x, t)=(\sin x)^{2}, x \in[0,1]$, corresponding to the DQM and FD (backward Euler method). The comparison between FD and quadrature results shows that the DQ method produces the same accuracy with much larger mesh size (about ten times larger) and the speed of the DQM is, hence, much faster than the FD methods.

\section{Discrete Modeling BY DQMS}

Assume that a multiconductor transmission line stretches from 0 to $d$ along the $z$-axis of a Cartesian coordinate system, where $d$ is the length of the line. Let $V(z, s)$ and $I(z, s)$ be, respectively, the Laplace-domain voltage and current vectors at point $z$, and let the distributed per-unit-length (PUL) parameter matrices of the line be denoted by $r(z), l(z), g(z)$, and $c(z)$, representing resistance, inductance, conductance, and capacitance, respectively. Use a linear transformation to normalize the line stretch $[0, d]$ to $[0,1]$ by setting $z=x d$. The normalized Telegrapher's equations can be written as

$$
\begin{aligned}
\frac{d}{d x} V(x, s) & =-(s L(x)+R(x)) I(x, s) \\
\frac{d}{d x} I(x, s) & =-(s C(x)+G(x)) V(x, s)
\end{aligned}
$$

where $R(x), L(x), G(x)$, and $C(x)$ are the normalized PUL parameters, which are obtained by multiplying $r(x), l(x), g(x)$, and $c(x)$, respectively, by the length of the transmission line $d$.

In order to concisely express some of the manipulations required in a matrix, the following terminologies are first defined here.

Definition 1: A discretization operator $\mathbf{D}$ discretizes a function $f(x)$ into a diagonal matrix whose entries are function values at the grid points $\left\{x_{1}, x_{2} \cdots x_{N}\right\}$

$$
\mathrm{D}(f)=\operatorname{diag}\left\{f\left(x_{1}\right), f\left(x_{2}\right), \ldots, f\left(x_{N}\right)\right\}
$$

Definition 2: Let $\mathbf{X}$ be an $m \times n$ matrix. A colon notation denotes that the specific rows and columns of a matrix are selected to define a submatrix. Thus, $\mathbf{X}_{i: j}$ is the $(j-i+1) \times n$ submatrix (between the $i$ th and $j$ th rows) of $\mathbf{X}$.

\section{A. Single Transmission Line}

At first, we will derive the discretized DQM model for a standalone single transmission line. Assume that the distributed voltage and current in (18) and (19) be interpolated and approximated by

$$
\begin{aligned}
V(x, s) & =\sum_{i=1}^{N} \chi_{i}(x) V_{i}(s) \\
I(x, s) & =\sum_{i=1}^{N} \chi_{i}(x) I_{i}(s)
\end{aligned}
$$

where $\chi_{i}(x)$ is the function like (8) or (13) and $V_{i}(s)=$ $V\left(x_{i}, s\right), I_{i}(s)=I\left(x_{i}, s\right)$.
By using (17), the frequency-domain Telegrapher's equations of a single lossy (RLCG) line are transformed into

$$
\left[\begin{array}{cc}
\mathbf{D}(G)+s \mathbf{D}(C) & \mathbf{A} \\
\mathbf{A} & \mathbf{D}(R)+s \mathbf{D}(L)
\end{array}\right]\left[\begin{array}{l}
\mathbf{V} \\
\mathbf{I}
\end{array}\right]=\mathbf{0}
$$

where

$$
\begin{aligned}
\mathbf{A} & =\left[a_{i j}\right] \in R^{N \times N} \\
\mathbf{V} & =\left[V_{1}(s), V_{2}(s), \ldots, V_{N}(s)\right]^{T} \\
\mathbf{I} & =\left[I_{1}(s), I_{2}(s), \ldots, I_{N}(s)\right]^{T}
\end{aligned}
$$

and the other matrices are defined by using (20) as follows:

$$
\begin{aligned}
& \mathrm{D}(L)=\operatorname{diag}\left\{L\left(x_{1}\right), L\left(x_{2}\right), \ldots, L\left(x_{N}\right)\right\} \\
& \mathrm{D}(R)=\operatorname{diag}\left\{R\left(x_{1}\right), R\left(x_{2}\right), \ldots, R\left(x_{N}\right)\right\} \\
& \mathrm{D}(C)=\operatorname{diag}\left\{C\left(x_{1}\right), C\left(x_{2}\right), \ldots, C\left(x_{N}\right)\right\} \\
& \mathrm{D}(G)=\operatorname{diag}\left\{G\left(x_{1}\right), G\left(x_{2}\right), \ldots, G\left(x_{N}\right)\right\}
\end{aligned}
$$

with the boundary points given by $x_{1}=0$ and $x_{N}=1$.

Thus, the Telegrapher's equations of a single transmission line are discretized to (23). However, as no excitations (boundary conditions) are involved in (23), it thereby has no nontrivial solutions, which is theoretically resulted from the properties of DQ operators (Lemmas 1-3). As in the cases with the FD method, here also, we can analogously replace the equations at an appropriate point within the range $\left\{x_{1}, x_{2}, \ldots, x_{N}\right\}$ by those at the boundary conditions. An alternative is to enforce (23), holding at each interior grid points $x_{i}, i=2, \ldots, N-1$, and to force the boundary conditions holding at the end points $x_{1}$ and $x_{N}$. The discretized Telegrapher's equations with boundary conditions can be written as follows:

$$
\left[\begin{array}{cc}
\mathbf{D}(G) & \mathbf{A} \\
\mathbf{A} & \mathbf{D}(R)
\end{array}\right]\left[\begin{array}{c}
\mathbf{V} \\
\mathbf{I}
\end{array}\right]+s\left[\begin{array}{cc}
\mathbf{D}(C) & \mathbf{0} \\
\mathbf{0} & \mathbf{D}(L)
\end{array}\right]\left[\begin{array}{c}
\mathbf{V} \\
\mathbf{I}
\end{array}\right]=\left[\begin{array}{c}
\mathbf{B}_{I} \\
\mathbf{B}_{V}
\end{array}\right]
$$

\section{B. Multiconductor Transmission Lines}

The above technique for a single transmission line can be now extended to model multiconductor transmission lines, where the conductors are closely spaced to include coupling effects between them. Assuming that the transmission lines are comprised of $M$-coupled interconnects that have a common reference, the Telegrapher's equations for an individual line in this case transform into (25) and (26) as follows:

$$
\begin{aligned}
\frac{d}{d x} V_{i}(x, s) & =-\sum_{j=1}^{M}\left[s L_{i j}(x)+R_{i j}(x)\right] I_{j}(x, s) \\
\frac{d}{d x} I_{i}(x, s) & =-\sum_{j=1}^{M}\left[s C_{i j}(x)+G_{i j}(x)\right] V_{j}(x, s) .
\end{aligned}
$$


In order to apply a DQM operator to every derivative, the distributed voltage and current along the line are, respectively, discretized as

$$
\begin{aligned}
\mathbf{V}_{i} & =\left[V_{i}\left(x_{1}, s\right), V_{i}\left(x_{2}, s\right), \ldots, V_{i}\left(x_{N}, s\right)\right]^{T} \\
\mathbf{I}_{i} & =\left[I_{i}\left(x_{1}, s\right), I_{i}\left(x_{2}, s\right), \ldots, I_{i}\left(x_{N}, s\right)\right]^{T}
\end{aligned}
$$

and the distributed parameters are discretized as

$$
\begin{aligned}
& \mathrm{D}\left(R_{i j}\right)=\operatorname{diag}\left\{R_{i j}\left(x_{1}\right), R_{i j}\left(x_{2}\right), \ldots, R_{i j}\left(x_{N}\right)\right\} \\
& \mathrm{D}\left(L_{i j}\right)=\operatorname{diag}\left\{L_{i j}\left(x_{1}\right), L_{i j}\left(x_{2}\right), \ldots, L_{i j}\left(x_{N}\right)\right\} \\
& \mathrm{D}\left(G_{i j}\right)=\operatorname{diag}\left\{G_{i j}\left(x_{1}\right), G_{i j}\left(x_{2}\right), \ldots, G_{i j}\left(x_{N}\right)\right\} \\
& \mathrm{D}\left(C_{i j}\right)=\operatorname{diag}\left\{C_{i j}\left(x_{1}\right), C_{i j}\left(x_{2}\right), \ldots, C_{i j}\left(x_{N}\right)\right\}
\end{aligned}
$$

each of which is an $N \times N$ matrix.

In a similar way to the single conductor, enforcing the boundary conditions at the end points $x_{1}$ and $x_{N}$, we can obtain the discretized Telegrapher's equations with boundary conditions for multiconductor transmission lines

$$
\left[\begin{array}{cc}
\hat{\mathbf{D}}(G) & \hat{\mathbf{A}} \\
\hat{\mathbf{A}} & \hat{\mathbf{D}}(R)
\end{array}\right]\left[\begin{array}{c}
\hat{\mathbf{V}} \\
\hat{\mathbf{I}}
\end{array}\right]+s\left[\begin{array}{cc}
\hat{\mathbf{D}}(C) & \mathbf{0} \\
\mathbf{0} & \hat{\mathbf{D}}(L)
\end{array}\right]\left[\begin{array}{c}
\hat{\mathbf{V}} \\
\hat{\mathbf{I}}
\end{array}\right]=\left[\begin{array}{c}
\hat{\mathbf{b}}_{I} \\
\hat{\mathbf{b}}_{V}
\end{array}\right]
$$

where

$$
\begin{aligned}
\hat{\mathbf{V}} & =\left[\mathbf{V}_{1} \cdots \mathbf{V}_{M}\right]^{T} \\
\hat{\mathbf{I}} & =\left[\mathbf{I}_{1} \cdots \mathbf{I}_{M}\right]^{T} \\
\hat{\mathbf{A}} & =\left[\begin{array}{ccc}
\mathbf{A} & \cdots & \mathbf{0} \\
\vdots & \ddots & \vdots \\
\mathbf{0} & \cdots & \mathbf{A}
\end{array}\right] \\
\hat{\mathbf{D}}(R) & =\left[\begin{array}{ccc}
\mathbf{D}\left(R_{11}\right) & \cdots & \mathbf{D}\left(R_{1 M}\right) \\
\vdots & \ddots & \vdots \\
\mathbf{D}\left(R_{M 1}\right) & \cdots & \mathbf{D}\left(R_{M M}\right)
\end{array}\right] \\
\hat{\mathbf{D}}(L) & =\left[\begin{array}{ccc}
\mathbf{D}\left(L_{11}\right) & \cdots & \mathbf{D}\left(L_{1 M}\right) \\
\vdots & \ddots & \vdots \\
\mathbf{D}\left(L_{M 1}\right) & \cdots & \mathbf{D}\left(L_{M M}\right)
\end{array}\right] \\
\hat{\mathbf{D}}(G) & =\left[\begin{array}{ccc}
\mathbf{D}\left(G_{11}\right) & \cdots & \mathbf{D}\left(G_{1 M}\right) \\
\vdots & \ddots & \vdots \\
\mathbf{D}\left(G_{M 1}\right) & \cdots & \mathbf{D}\left(G_{M M}\right)
\end{array}\right] \\
\hat{\mathbf{D}}(C) & =\left[\begin{array}{ccc}
\mathbf{D}\left(C_{11}\right) & \cdots & \mathbf{D}\left(C_{1 M}\right) \\
\vdots & \ddots & \vdots \\
\mathbf{D}\left(C_{M 1}\right) & \cdots & \mathbf{D}\left(C_{M M}\right)
\end{array}\right] .
\end{aligned}
$$

Inverse Laplace transforms of (24) and (27) lead to first-order ordinary differential equations, which represent the time-domain models of interconnects. The time-domain responses at the ends of transmission lines can be obtained by solving ordinary differential equations.
On the other hand, DQM modeling supplies general discrete models ready for the circuit reduction. In order to apply Krylov subspace techniques for the model order reduction, the coefficient matrices of the voltage and current variables must be first-degree polynomials in $s$ [21]. The first order with respect to $s$ in (24) and (27) allows to reduce a large subnetwork containing distributed transmission lines to a small macromodel.

\section{Compact Models Based on Discretization}

The admittance matrix model based on the discretized model can be obtained by calculating $I_{1}(s)$ and $I_{N}(s)$ while setting $V_{1}(s)=1$ and $V_{N}(s)=1$. The state equation takes the form

$$
(\mathbf{P}+s \mathbf{Q}) \mathbf{X}=\mathbf{B}
$$

where the matrices have the following form:

$$
\begin{aligned}
& \mathbf{X}=\left[\begin{array}{l}
\mathbf{V} \\
\mathbf{I}
\end{array}\right] \\
& \mathbf{P}=\left[\begin{array}{cc}
\mathbf{D}(G) & \mathbf{A} \\
\mathbf{A}^{\prime \prime} & \mathbf{D}^{\prime \prime}(R)
\end{array}\right] \\
& \mathbf{Q}=\left[\begin{array}{cc}
\mathbf{D}(C) & \mathbf{0} \\
\mathbf{0} & \mathbf{D}^{\prime \prime}(L)
\end{array}\right] \\
& \mathbf{B}=\left[\begin{array}{llllll}
0 & \cdots & 0 & 1 & \cdots & 0 \\
0 & \cdots & 0 & 0 & \cdots & 1
\end{array}\right]^{T}
\end{aligned}
$$

in which $\mathbf{D}^{\prime \prime}(L)$ and $\mathbf{D}^{\prime \prime}(R)$ are represented by

$$
\begin{aligned}
& \mathrm{D}^{\prime \prime}(L)=\operatorname{diag}\left\{0, L\left(x_{2}\right), \ldots, L\left(x_{N-1}\right), 0\right\} \\
& \mathrm{D}^{\prime \prime}(R)=\operatorname{diag}\left\{0, R\left(x_{2}\right), \ldots, R\left(x_{N-1}\right), 0\right\}
\end{aligned}
$$

and $\mathbf{A}^{\prime \prime}$ is a matrix generated by replacing the first and $N$ th row of matrix $\mathbf{A}$ with vector $\left\{\begin{array}{llll}1 & 0 \cdots 0\end{array}\right\}$ and $\left\{\begin{array}{lll}0 \cdots 0 & 1\end{array}\right\}$, respectively. Using the definition of colon notation, it is represented by

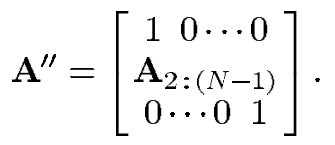

The admittance matrix follows:

$$
\mathbf{Y}=\mathbf{B}^{T}(\mathbf{P}+s \mathbf{Q})^{-1} \mathbf{B} .
$$

Similarly, each of the entries of admittance matrix is approximated by a rational formula, whose numerator and denominator have the same highest order, i.e., $(2 N-4)$ th.

Likewise, the admittance matrix of a multiconductor transmission line can be calculated. Derived from (27), the state equation in this case follows:

$$
(\hat{\mathbf{P}}+s \hat{\mathbf{Q}}) \hat{\mathbf{X}}=\hat{\mathbf{B}}
$$


where

$$
\begin{aligned}
\hat{\mathbf{X}} & =\left[\begin{array}{c}
\hat{\mathbf{V}} \\
\hat{\mathbf{I}}
\end{array}\right] \\
\hat{\mathbf{P}} & =\left[\begin{array}{cc}
\hat{\mathbf{D}}(G) & \hat{\mathbf{A}} \\
\hat{\mathbf{A}}^{\prime \prime} & \hat{\mathbf{D}}^{\prime \prime}(R)
\end{array}\right] \\
\hat{\mathbf{Q}} & =\left[\begin{array}{ccc}
\hat{\mathbf{D}}(C) & \mathbf{0} \\
0 & \hat{\mathbf{D}}^{\prime \prime}(L)
\end{array}\right] \\
\hat{\mathbf{B}} & =\left[\begin{array}{ccccccccc}
0 & \cdots & 0 & 1 & \cdots & 0 & 0 & \cdots & 0 \\
0 & \cdots & 0 & 0 & \cdots & 1 & 0 & \cdots & 0 \\
& \cdots & & & \cdots & & & \cdots & \\
0 & \cdots & 0 & 0 & \cdots & 0 & 1 & \cdots & 0 \\
0 & \cdots & 0 & 0 & \cdots & 0 & 0 & \cdots & 1
\end{array}\right]^{T}
\end{aligned}
$$

in which $\hat{\mathbf{A}}^{\prime \prime}, \hat{\mathbf{D}}^{\prime \prime}(L)$, and $\hat{\mathbf{D}}^{\prime \prime}(R)$ are defined accordingly [see (29)-(31)] as follows:

$$
\begin{aligned}
\hat{\mathbf{A}}^{\prime \prime} & =\left[\begin{array}{ccc}
\mathbf{A}^{\prime \prime} & \cdots & \mathbf{0} \\
\vdots & \ddots & \vdots \\
\mathbf{0} & \cdots & \mathbf{A}^{\prime \prime}
\end{array}\right] \\
\hat{\mathbf{D}}^{\prime \prime}(R) & =\left[\begin{array}{ccc}
\mathrm{D}^{\prime \prime}\left(R_{11}\right) & \cdots & \mathrm{D}^{\prime \prime}\left(R_{1 M}\right) \\
\vdots & \ddots & \vdots \\
\mathrm{D}^{\prime \prime}\left(R_{M 1}\right) & \cdots & \mathrm{D}^{\prime \prime}\left(R_{M M}\right)
\end{array}\right] \\
\hat{\mathbf{D}}^{\prime \prime}(L) & =\left[\begin{array}{ccc}
\mathrm{D}^{\prime \prime}\left(L_{11}\right) & \cdots & \mathrm{D}^{\prime \prime}\left(L_{1 M}\right) \\
\vdots & \ddots & \vdots \\
\mathrm{D}^{\prime \prime}\left(L_{M 1}\right) & \cdots & \mathrm{D}^{\prime \prime}\left(L_{M M}\right)
\end{array}\right] .
\end{aligned}
$$

Therefore, the admittance matrix for multiconductor transmission lines can be calculated by

$$
\hat{\mathbf{Y}}=\hat{\mathbf{B}}^{T}(\hat{\mathbf{P}}+s \hat{\mathbf{Q}})^{-1} \hat{\mathbf{B}}
$$

\section{Companion Models}

Circuit reduction can be performed on discrete models of a transmission line, as is done in much of the literature. Instead of following this approach, we have directly developed companion models from a discrete model. In order to handle nonlinear and time-varying systems, a time-domain model of each kind of device is needed. According to the literature [27], in order to achieve maximum efficiency in a reasonable time, the line simulation should be based on a device model that does not require the introduction of current variables. Considering the modified nodal analysis (MNA) matrix requirement, the admittance-formed model is preferred. In (32), each item in the admittance matrix is a rational formula having the order of its numerator and denominator $(n=(2 N-4))$ determined by the DQ order $N$. By using the technique of recursive convolution on (32), a companion model can be constituted.

For an example of a single transmission line, the admittance matrix in (32) can be represented by

$$
\mathbf{Y}=\left[\begin{array}{cc}
Y_{11}(s) & Y_{1 N}(s) \\
Y_{N 1}(s) & Y_{N N}(s)
\end{array}\right]
$$

where each of $Y_{11}, Y_{1 N}, Y_{N 1}$, and $Y_{N N}$ is a rational approximation whose numerator and denominator are both $(2 N-4)$ th-order polynomials. Let us consider $Y_{11}(s)$. Applying Heaviside's theorem, it can be decomposed in the following form:

$$
Y_{11}(s)=\frac{\sum_{k=1}^{n} a_{k} s^{k}}{\sum_{k=1}^{n} b_{k} s^{k}}=\frac{a_{n}}{b_{n}}+\sum_{k=1}^{n} \frac{q_{k}}{s-p_{k}} .
$$

The inverse Laplace transform of (36) is

$$
y_{11}(t)=\frac{a_{n}}{b_{n}} \delta(t)+\sum_{k=1}^{n} q_{k} \exp \left(p_{k} t\right)
$$

and the other entries $Y_{1 N}(s), Y_{N 1}(s)$, and $Y_{N N}(s)$ can be similarly formulated. Thus, taking the inverse Laplace transform of (35), we can obtain the time-domain counterparts

$$
\begin{gathered}
i_{1}(t)=y_{11}(t) * v_{1}(t)+y_{1 N}(t) * v_{N}(t) \\
i_{N}(t)=y_{N 1}(t) * v_{1}(t)+y_{N N}(t) * v_{N}(t)
\end{gathered}
$$

where “ $*$ " denotes the convolution operation, and $v_{1}(t), v_{N}(t)$, $i_{1}(t)$, and $i_{N}(t)$ are, respectively, the time-domain counterparts of $V_{1}(s), V_{N}(s), I_{1}(s)$, and $I_{N}(s)$.

As $y_{11}(t)$ is cast in exponential form, the convolution integration in (38) and (39) can be calculated by applying the recursive convolution [10], and the companion model can be developed, which has linear complexity with respect to the simulation time. Similarly, the companion model of multiconductor transmission lines can also be derived.

\section{ORDER AND ACCURACY OF DQM MODELING}

A good way to determine the order and accuracy of the DQM is to apply the Chebyshev pseudospectral expansion [28], [29]. According to [29], the maximum frequency of interest can be evaluated by

$$
f_{\max }=\frac{1}{t_{r}}
$$

where $t_{r}$ is the rise time of the input waveform. The maximum frequency determines the minimum wavelength within the spectral range of interest. A heuristic rule for satisfying the resolution requirements of Chebyshev expansions is to use at least four collocation points per wavelength [29], and a resolution of two points per wavelength is sufficient for the modified Chebyshev method [28].

It is significant that the Chebyshev expansion method is, in fact, equivalent to the DQM in case its coefficients are determined by Chebyshev polynomials [called Chebyshev differential quadratures (CDQs)]. Therefore, the criteria for the selection of collocation points are basically applicable to CDQ. However, as the order of Chebyshev expansion increases, the collocation points of the zeros of Chebyshev polynomials tend to concentrate at two ends of the line. For the nonuniform transmission line, it leads to an oversampling of the voltages and currents at the end points of the line and an 


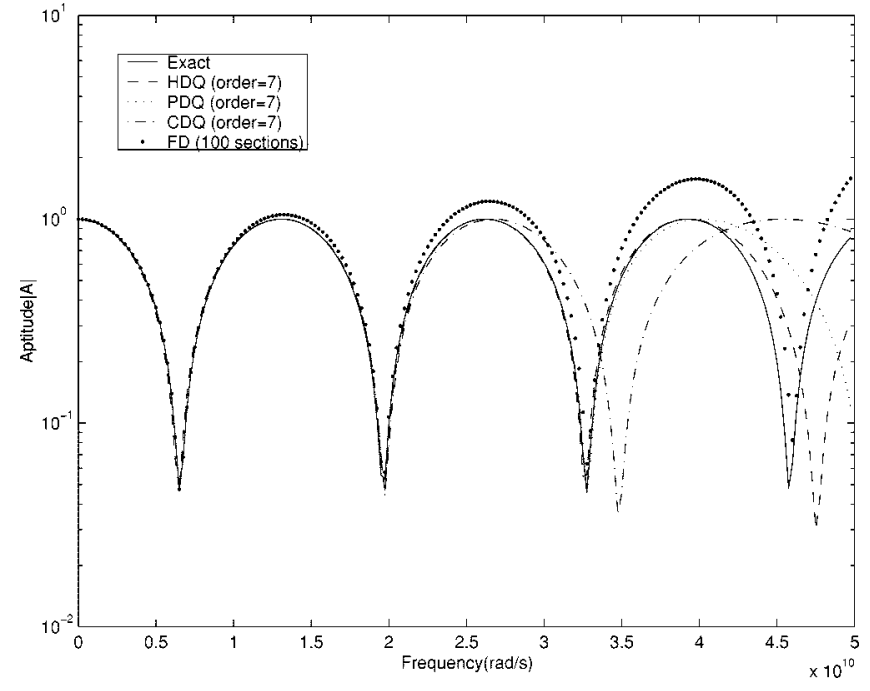

Fig. 1. Frequency responses by DQM and FD modeling.

undersampling at the center segment of the line. The crowded points at the boundaries and the sparse points at the center will cause significant loss of accuracy when handling a nonuniform transmission line.

On the other hand, DQM with coefficients determined by harmonic functions (called HDQs), overcomes this difficulty. The grid points of an HDQ need not be equally spaced, and thereby can be discreetly selected by the user. In this paper, in order to determine the HDQ coefficients, we select the collection of points that are equally spaced over the entire length of the transmission line. In this way, the coefficients can be completely determined as fixed constants and can be applied to any problem in general.

In order to verify the accuracy of the DQ method, a practical example is discussed below. A part of a GaAs/InP interconnect used by the authors and their co-researchers to design ultrafast circuits [30] is modeled as a single transmission line. The PUL parameters are extracted by the methods illustrated in [31] and are set to $l=360 \mathrm{nH} / \mathrm{m}, c=100 \mathrm{pF} / \mathrm{m}, r=100 \Omega / \mathrm{m}$, and $g=0.01 \mathrm{~S} / \mathrm{m}$. The line length is assumed to be $4 \mathrm{~cm}$. By employing the abovementioned procedure, the transmission line can be represented as an equivalent two-port model.

Fig. 1 shows the frequency-domain response of the voltage transfer function of the two-port model. It is noted that the HDQ gives higher accuracy than other methods, and the accuracy of the conventional FD method is the lowest. A heuristic rule for the resolution of the HDQ is to collect two points per minimum wavelength in the spectrum

$$
N=2 \frac{d}{\lambda_{\min }}+2
$$

By this rule, the seventh-order HDQ can guarantee high accuracy within the frequency band from zero to more than 40 $\mathrm{Grad} / \mathrm{s}$, as shown in Fig. 1. That means, besides the two end points, only five inner points along the transmission line are needed to obtain the accuracy in Fig. 1, while the FD method cannot guarantee accuracy even if 100 grid points are sampled. It is also shown that the accuracy of the HDQ is more accurate
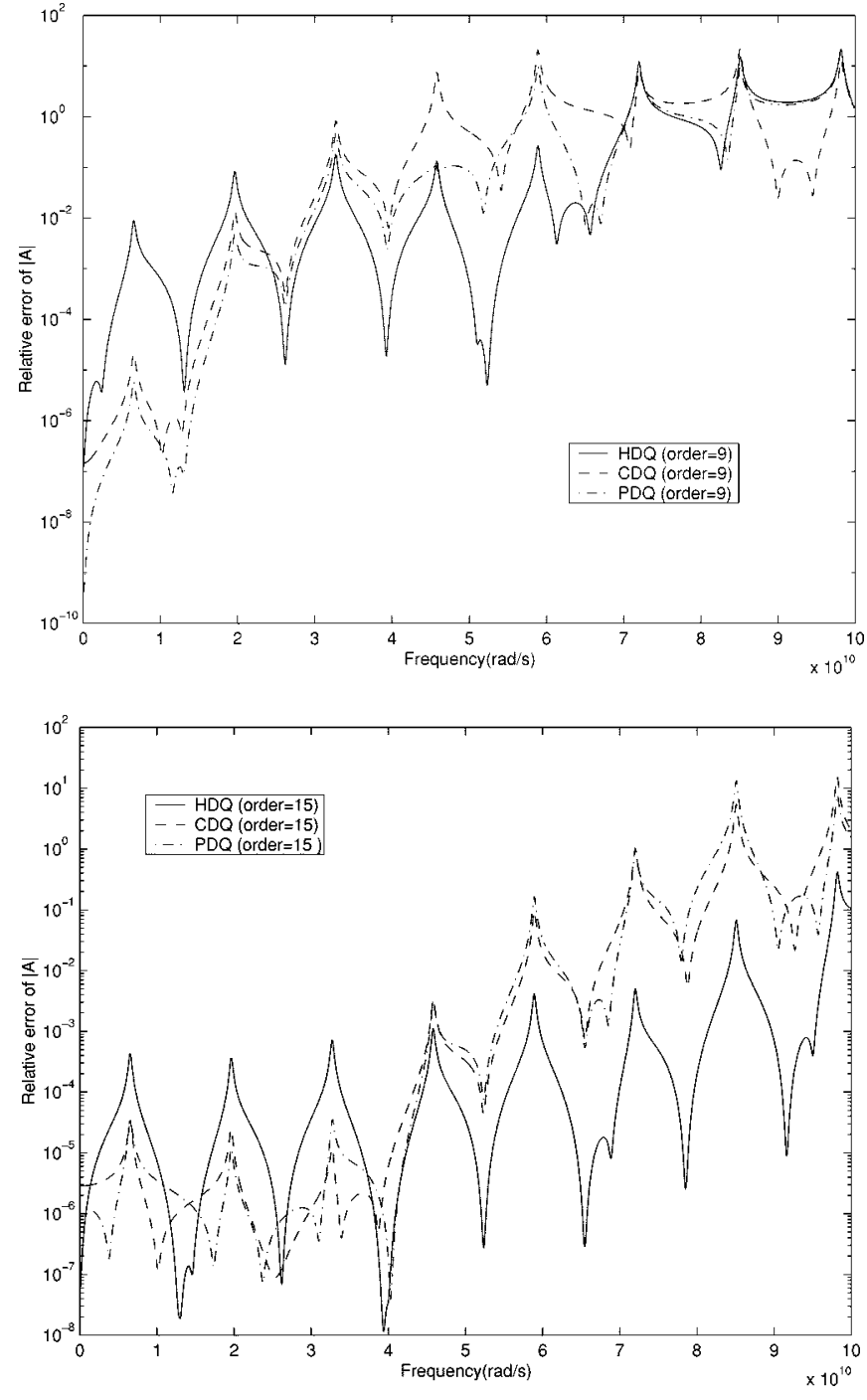

Fig. 2. Relative errors of ninth- and fifteenth-order DQM modelings.

than that of any other DQM with the same order. The relative errors of a higher order HDQ, CDQ, and PDQ are shown in Fig. 2.

\section{Circuit Formulation AND APPliCATIONS}

The model presented in the previous sections can be directly incorporated in formulating circuit models in the frequency domain, as well as in the time domain. Consider a linear circuit net that contains linear lumped components and multiconductor transmission-line systems. Without loss of generality, the timedomain MNA matrix equations for the circuit net with an impulse excitation as its source input can be written as [32]

$$
\mathbf{C}_{\text {net }} \frac{d}{d t} \mathbf{v}_{\text {net }}(t)+\mathbf{G}_{\text {net }} \mathbf{v}_{\text {net }}(t)+\sum_{k=1}^{N_{s}} \mathbf{P}_{k} \mathbf{i}_{k}(t)=\mathbf{B} u(t)
$$

where $\mathbf{v}_{\text {net }}(t) \in R^{n}$ is a vector containing the node voltages, independent voltage source, and all other additional variables, $\mathbf{B} \in R^{n}, \mathbf{B} u(t)$ is a vector representing the excitations from the independent source, $\mathbf{C}_{\text {net }} \in R^{n \times n}$ and $\mathbf{G}_{\text {net }} \in R^{n \times n}$ are constant matrices formed by linear lumped components of net, $\mathbf{P}_{k} \in R^{n \times n_{k}}$, which has entries $p_{i j} \in\{0,1\}$, is a selector 


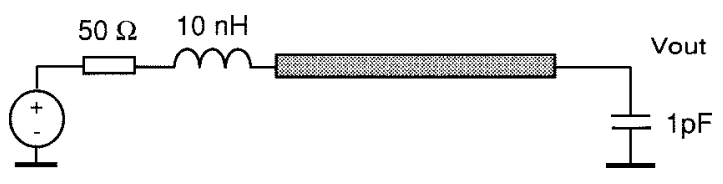

Fig. 3. Single transmission line.

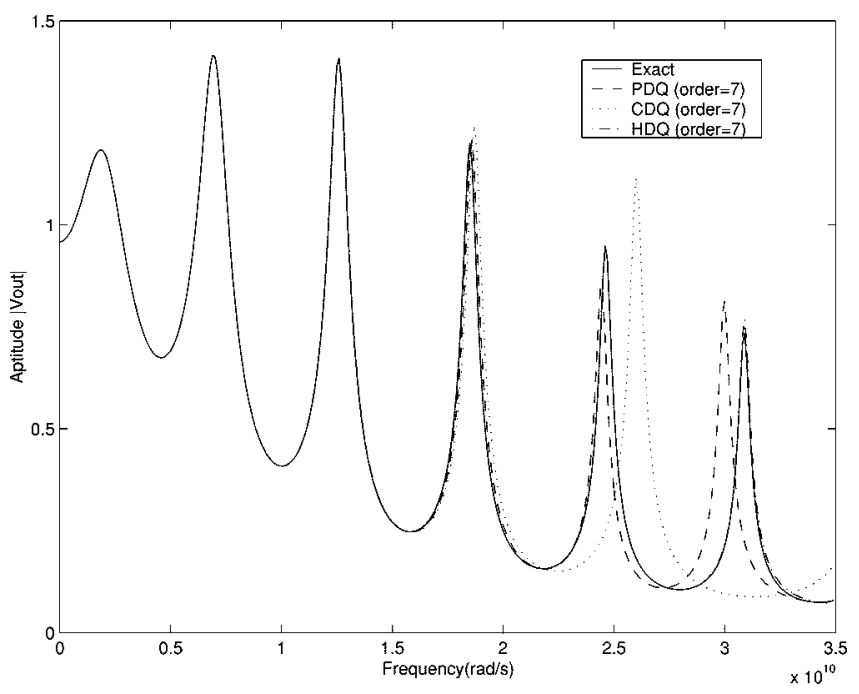

Fig. 4. Frequency responses by different DQMs.

matrix; it maps $\mathbf{i}_{k}(t)$, the terminal currents of the line system or additional variables into the node space of the circuit net, and $N_{s}$ is the number of subnet.

The DQM models of transmission lines described by (24) and (27) or their companion models can be incorporated into (42) as an MNA stamp. We next present several examples. All the PDQs and HDQs used in these examples employ equally spaced grid points, and the CDQ employ Chebyshev grid points. Since the distributions of the grid points have been fixed, all the DQ coefficient matrix used in these examples are available constants, which have been already obtained by the approaches of Section II.

The first example is a single transmission line, as shown in Fig. 3. The length of this transmission line is $8 \mathrm{~cm}$, and the PUL parameters are $l=360 \mathrm{nH} / \mathrm{m}, c=100 \mathrm{pF} / \mathrm{m}, r=100 \Omega / \mathrm{m}$, and $g=0.01 \mathrm{~S} / \mathrm{m}$. The applied input is a step voltage whose rise time is $0.2 \mathrm{~ns}$.

The propagation velocity along the line is $5 / 3 \times 10^{8} \mathrm{~m} / \mathrm{s}$, and by (40), the maximum frequency $f_{\max }=5 \mathrm{GHz}$. By applying (41), the order of the DQM is calculated as seven. Therefore, we employ the seventh-order HDQ, CDQ, and PDQ, respectively, to compute the frequency responses of the transmission line, as shown in Fig. 4. Obviously, the result of the HDQ is most agreeable with the exact value.

The transient responses of the transmission line corresponding to HDQ, CDQ, and PDQ modeling are shown in Fig. 5 along with the response obtained using the commercial simulator HSPICE. The transient result of the HDQ is more agreeable with that of HSPICE than the other two types of DQMs investigated in this paper. HSPICE employs a multiple lumped-filter section to generate the transient behavior in the time domain [33]. From these empirical studies, it may be noted that, compared to other numerical methods, the low-order

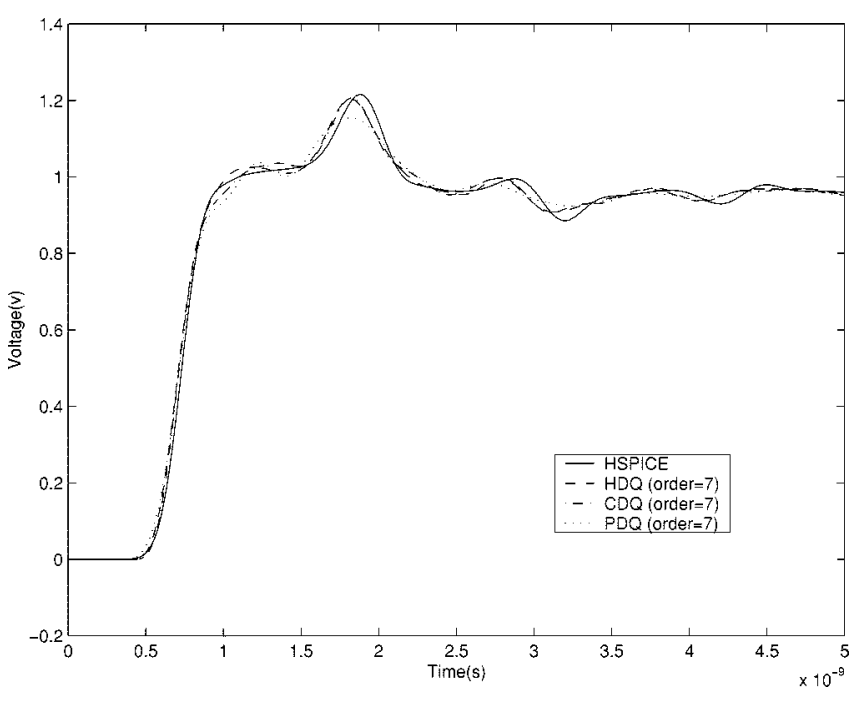

Fig. 5. Step response for a single transmission line.

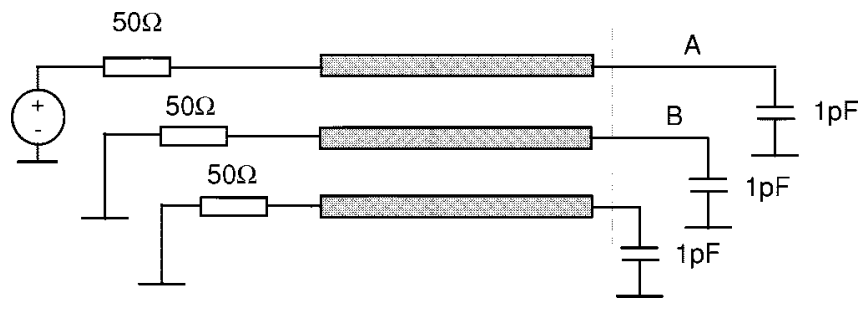

Fig. 6. Circuit of a coupled transmission line.

DQ method can give accurate transient results. In this example, the DQ method samples only five inner grid points along the transmission line. If the FD method is employed to solve the example, at least between 50 100 grid points are needed to achieve the same accuracy.

The second example we study in this paper consists of three coupled transmission lines, as shown in Fig. 6. The length of each transmission line is $5 \mathrm{~cm}$ and its RLGC parameters are represented in the following matrices:

$$
\begin{aligned}
& \mathbf{R}=\left[\begin{array}{ccc}
3.448 & 0 & 0 \\
0 & 3.448 & 0 \\
0 & 0 & 3.448
\end{array}\right] \Omega / \mathrm{cm} \\
& \mathbf{L}=\left[\begin{array}{ccc}
4.976 & 0.765 & 0 \\
0.765 & 4.976 & 0.765 \\
0 & 0.765 & 4.976
\end{array}\right] \mathrm{nH} / \mathrm{cm} \\
& \mathbf{C}=\left[\begin{array}{ccc}
1.082 & -0.197 & 0 \\
-0.197 & 1.124 & -0.197 \\
0 & -0.197 & 1.082
\end{array}\right] \mathrm{pF} / \mathrm{cm} \\
& \mathbf{G}=\mathbf{0} .
\end{aligned}
$$

Since the HDQ yields results closer to HSPICE, in this example of three coupled transmission lines, the HSPICE result is compared with the results of seventh-, ninth-, and eleventh-order HDQs. These correspond to deriving the harmonic coefficients by sampling seven, nine, and 11 equally spaced grid points on the transmission lines. The transient responses of point $\mathrm{A}$ are shown in Fig. 7, those of point B are shown in Fig. 8, and HDQ 


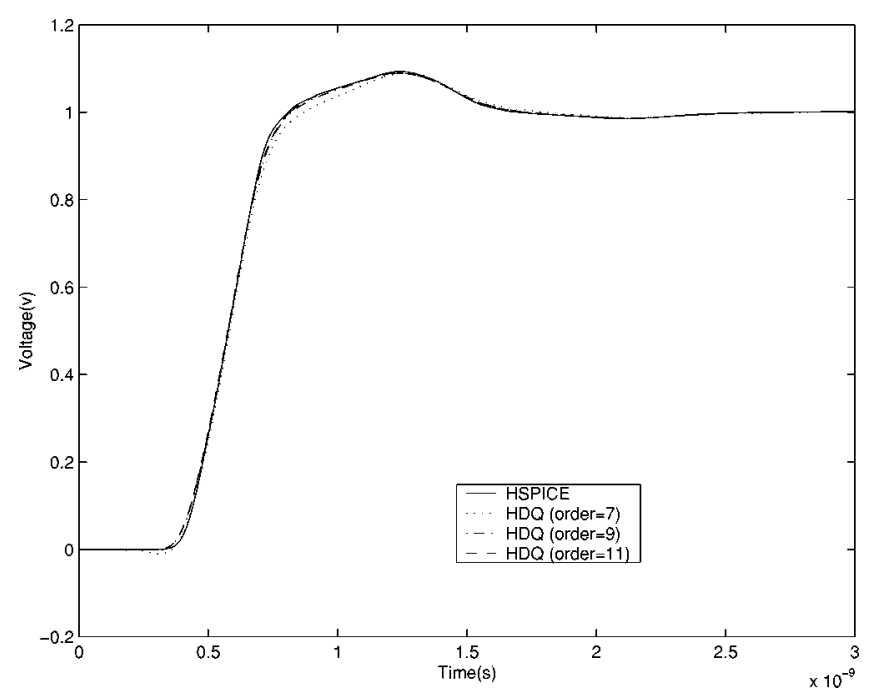

Fig. 7. Transient responses at $\mathrm{A}$ of a coupled transmission line.

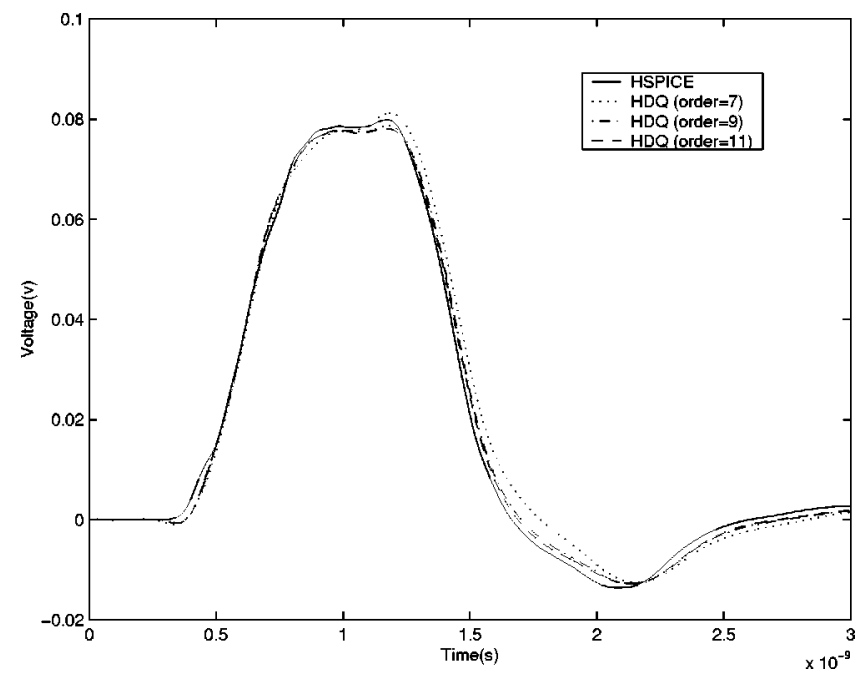

Fig. 8. Transient responses at $\mathrm{B}$ of a coupled transmission line.

results are compared with HSPICE results. It shows that the ninth-order DQ method gives more accurate results than the seventh-order DQ method. Generally, a higher order DQ method can give more accurate results. However, a higher order DQ means sampling of more points within the transmission line and more computation time for DQ coefficients. In practical applications, the DQ order can be selected no greater than 11. In our experiments, we found that the selection of a DQ order within the recommended range yields considerable accuracy while maintaining high computational efficiency.

One of the advantages of the proposed DQ method is that the technique can be applied without any difficulty to solve the multiconductor nonuniform transmission lines. We next study the third example of three lossy $(G>0)$ and coupled nonuniform transmission lines, as shown in Fig. 9. Since the width of the lines are assumed to vary across the length of transmission

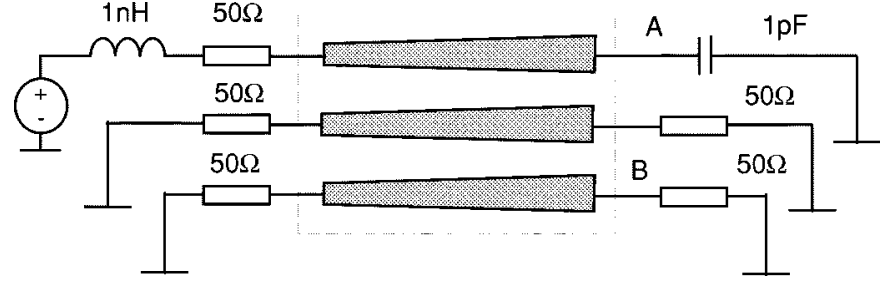

Fig. 9. Circuit of nonuniform interconnects.
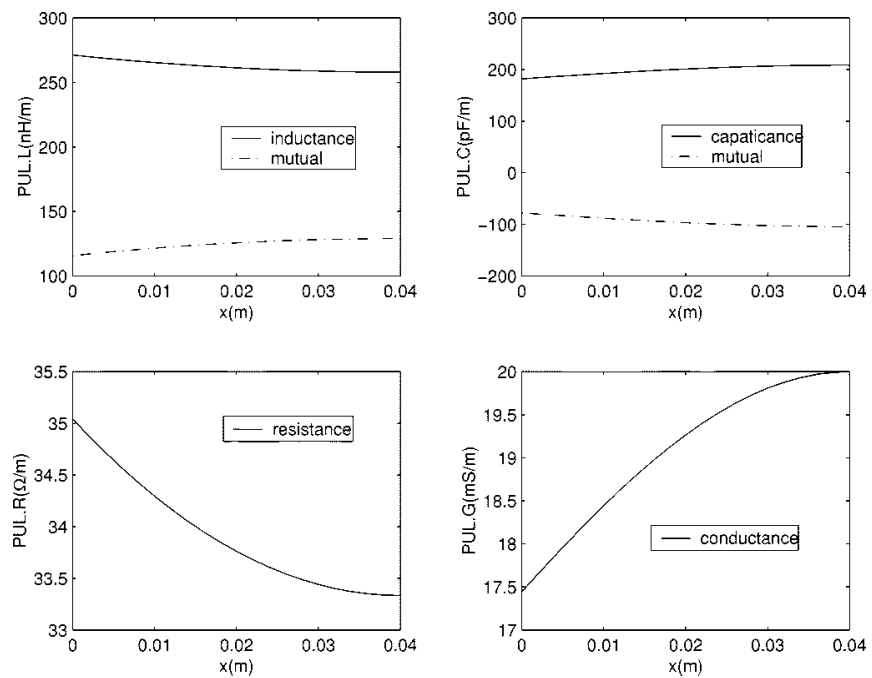

Fig. 10. Nonuniformity of the transmission line.

lines, the PUL parameters of the nonuniform transmission line can be represented as follows:

$$
\begin{aligned}
\mathbf{L} & =\left[\begin{array}{ccc}
L(x) & \operatorname{Lm}(x) & 0 \\
L m(x) & L(x) & L m(x) \\
0 & L m(x) & L(x)
\end{array}\right] \\
\mathbf{C} & =\left[\begin{array}{ccc}
C(x) & C m(x) & 0 \\
C m(x) & C(x) & C m(x) \\
0 & C m(x) & C(x)
\end{array}\right] \\
\mathbf{R} & =\left[\begin{array}{ccc}
R(x) & 0 & 0 \\
0 & R(x) & 0 \\
0 & 0 & R(x)
\end{array}\right] \\
\mathbf{G} & =\left[\begin{array}{ccc}
G(x) & 0 & 0 \\
0 & G(x) & 0 \\
0 & 0 & G(x)
\end{array}\right]
\end{aligned}
$$

where

$$
\begin{aligned}
L(x) & =387 /((1+k(x)) \mathrm{nH} / \mathrm{m} \\
L m(x) & =k(x) L(x) \\
C(x) & =104.3 /(1-k(x)) \mathrm{pF} / \mathrm{m} \\
C m(x) & =-k(x) C(x) \\
R(x) & =50 /(1+k(x)) \Omega / \mathrm{m} \\
G(x) & =0.01 /(1-k(x)) \mathrm{S} / \mathrm{m} \\
k(x) & =0.25(1+\sin (6.25 \pi x+0.25 \pi)) .
\end{aligned}
$$

The nonuniformity of the transmission line can be shown as Fig. 10. 


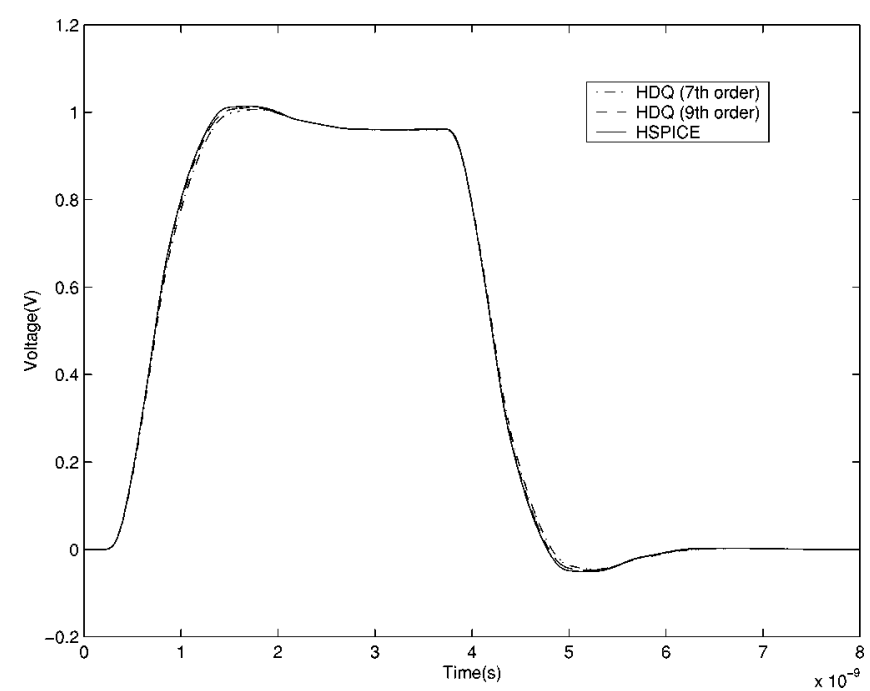

Fig. 11. Transient response at A of nonuniform interconnects.

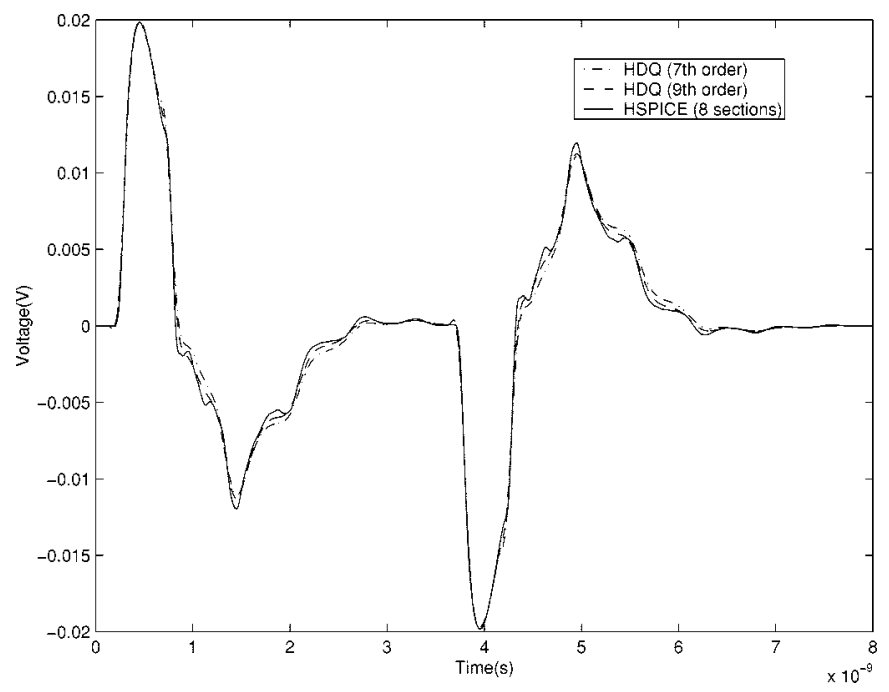

Fig. 12. Transient response at B of nonuniform interconnects.

The excitation is a 3-ns pulse with rise/fall times of $0.5 \mathrm{~ns}$. With seventh- and ninth-order harmonic coefficients, the transient responses of points A and B as computed by the HDQ are shown in Figs. 11 and 12. In order to verify the accuracy and efficiency of the DQ method, we apply HSPICE to this example. Since the nonuniform transmission line cannot be handled by HSPICE directly, the nonuniform line can only be incorporated into HSPICE in an indirect and inefficient way. We segment the lines into eight equal sections, each of which is regarded as a uniform line. In Figs. 11 and 12, the results of the DQ method and HSPICE are shown to match well, but the efficiencies of these two approaches are quite different. Since the time step of HSPICE is subject to the least flight delay of all the transmission lines, it cannot be too large to achieve an accurate transient result. In this example, HSPICE generates the indicated response in $5.527 \mathrm{~s}$ using a time step of $7 \mathrm{ps}$, while the HDQ method needs only $1.56 \mathrm{~s}$ to yield the similar response. The above computation times are based on the programs running on s Sun Ultra-1 workstation and do not include the time pertaining to reading and writing of files.

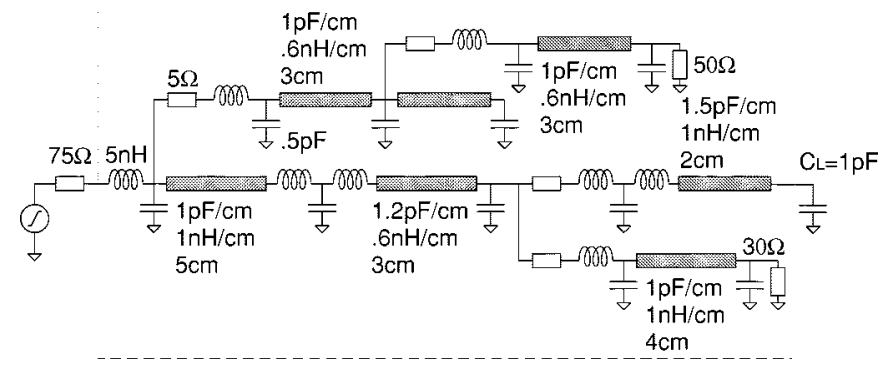

Fig. 13. Interconnect network.

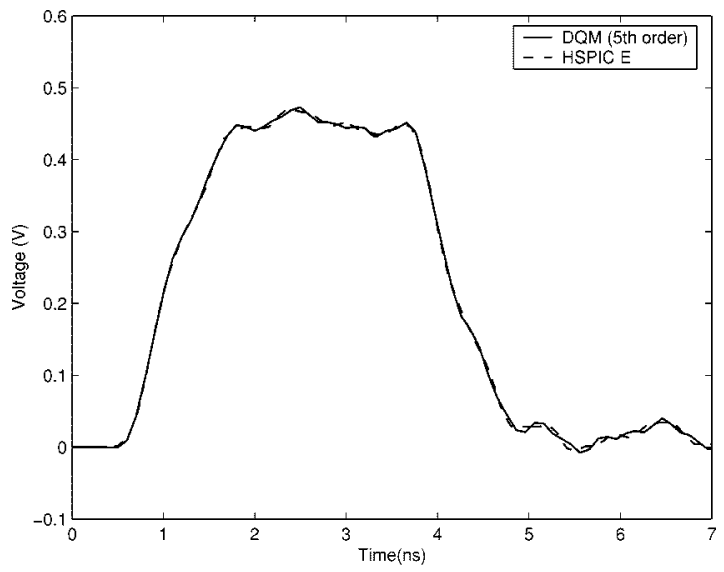

Fig. 14. Transient response of example 4.

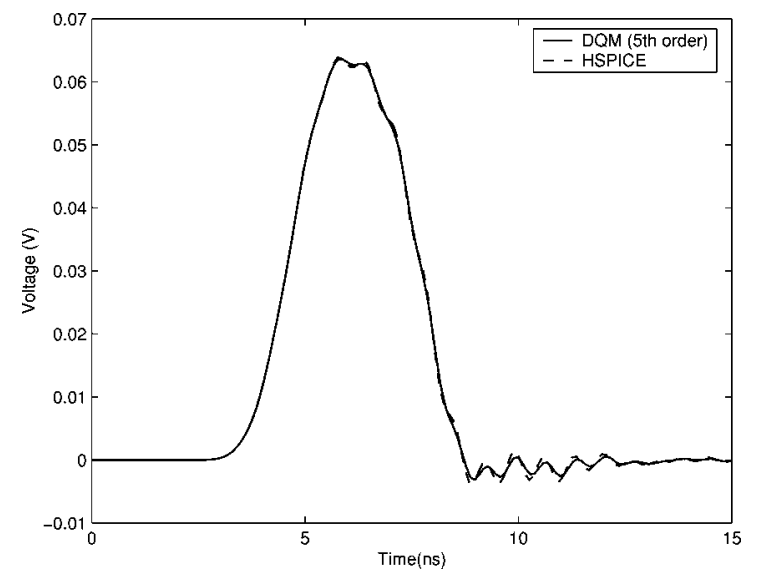

Fig. 15. Transient response of example 5.

The fourth example (see Fig. 13) shows a part of an MCM circuit containing $R L C$ lumped elements and distributed interconnects. For each of the interconnects in Fig. 13, the distributed resistance is $R=100 \Omega / \mathrm{m}$ and $G=0$. The input is an impulse with 3-ns width and 0.1-ns rise/fall time. By using a fifth-order PDQ model to represent the interconnects, the transient results are shown in Fig. 14 compared to the results of the FD model from HSPICE. On a Sun Ultra-1 workstation, the transient time of the DQM is $0.38 \mathrm{~s}$, while that of HSPICE is $1.44 \mathrm{~s}$.

The fifth example is to further test the efficiency of the DQM. The circuit is constructed by cascading five identical cells, each of which is the circuit shown in the dashed frame of Fig. 13. The transient responses in Fig. 15 are calculated by using a fifth-order PDQ model, as well as an HSPICE model. On the 
same Sun workstation, the transient time of the DQM is $3.44 \mathrm{~s}$, compared to the transient time of HSPICE, i.e., $10.84 \mathrm{~s}$.

\section{CONCLUSIONS}

In this paper, we have derived new transmission-line models by employing a powerful numerical computation technique, called the DQM. Unlike the FD and FE techniques, which compute functions and their derivatives over a cluster of locally neighboring points, the DQM discretizes the transmission line into few grid points across the entire length of the transmission line and computes the electrical parameters at those points in order to derive transient response of the transmission line. Based on the discrete models, compact models can be obtained, which take the form of rational approximations in the frequency domain and, thus, the companion model in the time domain can be obtained by an inverse Laplace transform and recursive convolution. Although the rational approximations of DQM models in the frequency domain are like the results of Padé approximation of AWE, the DQM solution procedure differs significantly from the AWE algorithm. One notable feature of the DQM is that it is more stable than AWE since it avoids the moment generation and moment-matching process altogether.

We have demonstrated how to compute DQ coefficients using harmonic functions and polynomials. Other techniques like the shifted Legendre polynomial, spline fitting, etc. can be studied and compared with the existing methods. In practical application, all the DQ coefficient matrices are fixed constants, which have been readily calculated prior to any modeling process. From our study with three DQ methods, we observe that the HDQ method, where DQ coefficients are determined by harmonic functions, yields the most accurate results. An $s$-domain DQM-based discrete model has matrix representation whose elements are first degree with respect to $s$, which is compatible with Krylov subspace techniques for circuit reduction. The companion model derived from the discrete model can be directly incorporated into circuit simulators such as SPICE. The HDQ has been shown to produce highly accurate delay models for both single and multiconductor transmission lines, having both uniform and nonuniform shapes. Unlike commercial simulators, which cannot directly handle nonuniform transmission lines, DQMs model the nonuniform lines by using the same procedure as model uniform lines and, therefore, at the same computational cost. Despite the fact that the DQM is a direct numerical computation technique, it remains stable and generates solutions faster than HSPICE (about three times) and many other well-known techniques.

\section{ACKNOWLEDGMENT}

The authors would like to acknowledge the helpful suggestions from Prof. Kuh, University of California at Berkeley.

\section{REFERENCES}

[1] A. Deutsch, "Electrical characteristics of interconnections for high-performance systems," Proc. IEEE, vol. 86, pp. 315-355, Feb. 1998.

[2] A. R. Djordjevic, T. K. Sarkar, and R. F. Harrington, "Time-domain response of multiconductor transmission lines," Proc. IEEE, vol. 75, pp. 743-764, June 1987.
[3] L. T. Pillage and R. A. Rohrer, "Asymptotic waveform evaluation for timing analysis," IEEE Trans. Computer-Aided Design, vol. 9, pp. 352-377, Apr. 1990.

[4] T. K. Tang and M. S. Nakhla, "Analysis of high-speed VLSI interconnect using the symptotic waveform evaluation technique," IEEE Trans. Computer-Aided Design, vol. 39, pp. 341-352, Mar. 1992.

[5] E. Chiprout and M. S. Nakhla, "Analysis of interconnect networks using complex frequency hopping," IEEE Trans. Computer-Aided Design, vol. 14, pp. 186-200, Feb. 1995.

[6] R. Achar, M. S. Nakhla, and Q.-J. Zhang, "Full-wave analysis of highspeed interconnects using complex frequency hopping," IEEE Trans. Computer-Aided Design, vol. 17, pp. 997-1015, Oct. 1998.

[7] A. B. Khang and S. Muddu, "Two-pole analysis of interconnection trees," in Proc. IEEE MCMC Conf., 1995, pp. 105-110.

[8] Q. Yu, J. M. L. Wang, and E. S. Kuh, "Passive multipoint moment matching model order reduction algorithm on multiport distributed interconnect networks," IEEE Trans. Circuits Syst. I, vol. 46, pp. 140-160, Jan. 1999.

[9] F. Y. Chang, "Transient analysis of lossy transmission lines with arbitrary initial potential and current distributions," IEEE Trans. Circuits Syst. I, vol. 39, pp. 180-198, Mar. 1992.

[10] S. Lin and E. S. Kuh, "Transient simulation of lossy interconnects based on the recursive convolution formulation," IEEE Trans. Circuits Syst. I, vol. 39, pp. 879-891, Nov. 1992.

[11] E. C. Chang and S.-M. Kang, "Transient simulation of lossy coupled transmission lines using iterative linear least square fitting and piecewise recursive convolution," IEEE Trans. Circuits Syst. I, vol. 43, pp. 923-932, Nov. 1996.

[12] F. Y. Chang, "The generalized method of characteristics for waveform relaxation analysis of lossy coupled transmission lines," IEEE Trans. Microwave Theory Tech., vol. 37, pp. 2028-2038, Dec. 1989.

[13] P. Feldman and R. W. Freund, "Efficient linear circuit analysis by Padé approximation via the Lanczos process," IEEE Trans. Computer-Aided Design, vol. 14, pp. 639-649, May 1995.

[14] _ - "Reduced-order modeling of large linear subcircuits via a block Lanczos algorithm," in Proc. 32nd ACM/IEEE Design Automation Conf., 1995, pp. 376-380.

[15] K. J. Kerns and A. T. Yang, "Stable and efficient reduction of large, multiport $R C$ networks by pole analysis via congruence transformations," IEEE Trans. Computer-Aided Design, vol. 16, pp. 734-744, July 1997.

[16] A. Odabasioglu, M. Celik, and L. T. Pileggi, "PRIMA: Passive reducedorder interconnect macromodeling algorithm," IEEE Trans ComputerAided Design, vol. 17, pp. 645-653, Aug. 1998.

[17] T. Dhaene and D. De Zutter, "Selection of lumped element models for coupled lossy transmission lines," IEEE Trans. Computer-Aided Design, vol. 11, pp. 805-815, July 1992.

[18] A. C. Cangellaris, M. Celik, S. Pasha, and L. Zhao, "Electromagnetic model order reduction for system-level modeling," IEEE Trans. Microwave Theory Tech., vol. 47, pp. 840-849, June 1999.

[19] B. Fornberg, A Practical Guide to Pseudospectral Methods. Cambridge, U.K.: Cambridge Univ. Press, 1996.

[20] C. Canuto, Spectral Methods in Fluid Dynamics. New York: SpringerVerlag, 1988.

[21] M. Celik and A. C. Cangellaris, "Simulation of multiconductor transmission lines using Krylov subspace order-reduction techniques," IEEE Trans. Computer-Aided Design, vol. 16, pp. 485-496, May 1997.

[22] R. Bellman, B. G. Kashef, and J. Casti, "Differential quadrature: A technique for the rapid solution of nonlinear partial differential equations," J. Comput. Phys., vol. 10, no. 1, pp. 40-52, 1972.

[23] C. Shu, "On the equivalence of generalized differential quadrature and highest order finite difference scheme," Comput. Methods Appl. Mech. Eng., vol. 155, pp. 249-260, 1998.

[24] R. Bellman and J. Casti, "Differential quadrature and long-term integration," J. Math. Anal. Applicat., vol. 34, no. 2, pp. 235-238, 1971.

[25] C. Shu, "Application of generalized differential quadrature to solve twodimensional incompressible Navier-Stokes equations," Int. J. Numer. Methods Fluids, vol. 15, no. 7, pp. 791-798, 1992.

[26] N. Bellomo, "Nonlinear models and problems in applied sciences from differential quadrature to generalized collocation methods," Math. Comput. Modeling, vol. 26, no. 4, pp. 13-34, 1997.

[27] D. B. Kuznetsov and J. E. Schutt-Aine, "Optimal transient simulation of transmission lines," IEEE Trans. Circuits Syst. I, vol. 43, pp. 110-121, Feb. 1996.

[28] D. Kosloff and H. Tal-Ezer, "A modified Chebyshev Pseudospectral method with an $O\left(N^{-1}\right)$ time step restriction," J. Comput. Phys., vol. 104, pp. 504-509, 1993. 
[29] R. Gupta, S.-Y. Kim, and L. T. Pillegi, "Domain characterization of design," IEEE Trans. Computer-Aided Design, vol. 16, pp. 184-193, Feb. 1996.

[30] P. Mazumder, S. Kulkarni, M. Bhattacharya, J. P. Sun, and G. I. Haddad, "Digital circuit applications of resonant tunneling devices," Proc. IEEE, vol. 86, pp. 664-686, Apr. 1998.

[31] A. K. Goel, High-Speed VLSI Interconnections: Modeling, Analysis, and Simulation. New York: Wiley, 1994.

[32] C. W. Ho, A. E. Ruehli, and P. A. Brennan, "The modified nodal approach to network analysis," IEEE Trans. Circuits Syst., vol. 22, pp. 504-509, June 1975.

[33] Star-HSPICE Manual, Avant! Corporation, Fremont, CA, 2000.

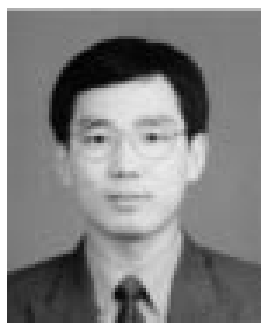

Qinwei Xu (S’02) was born in Shandong, China. He received the B.S. and M.S. degrees in electrical engineering from Shandong University, Shandong, China, in 1991 and 1994, respectively, and is currently working toward the Ph.D. degree in computer science and engineering at The University of Michigan at Ann Arbor.

His research interests include interconnect modeling, circuit simulation, and VLSI physical design.

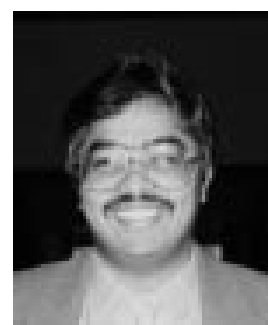

Pinaki Mazumder (S'84-M'88-SM'94-F'99) received the B.S.E.E. degree from the Indian Institute of Science, Bangalore, India, in 1976, the M.Sc. degree in computer science from the University of Alberta, Edmonton, AB, Canada, in 1985, and the Ph.D. degree in electrical and computer engineering from the University of Illinois at Urbana-Champaign, in 1987.

$\mathrm{He}$ is currently with the Department of Electrical Engineering and Computer Science, The University of Michigan at Ann Arbor. Prior to this, he spent two years as a Research Assistant with the Coordinated Science Laboratory, University of Illinois at Urbana-Champaign and over six years with Bharat Electronics Ltd. (a collaborator of RCA), Bangalore, India, where he developed several types of analog and digital integrated circuits for consumer electronics products. During the summer of 1985 and 1986, he was a Member of the Technical Staff of the Indian Hill Branch, AT\&T Bell Laboratories. From 1996 to 1997 , he spent his sabbatical leave as a visiting faculty member with Stanford University, University of California at Berkeley, and Nippon Telephone and Telegraph. His research interests include VLSI testing, physical design automation, and ultrafast circuit design. He has authored or coauthored over 160 papers on these topics in archival journals and proceedings of international conferences. He coauthored Testing and Testable Design of High-Density Random-Access Memories (Boston, MA: Kluwer, 1996), Semiconductor Memories: Testing and Reliability (Los Alamitos, CA: IEEE Comput. Soc., 1998), and Genetic Algorithms for VLSI Design, Layout, and Test Automation (Englewood Cliffs, NJ: Prentice-Hall, 1998). He has two international patents pending. He has lead his research group's efforts in VLSI testing and built-in self-repair techniques and has developed silicon compilers for RAM, ROM, and programmable logic array (PLA) with built-in self-repairable capabilities. He has also done extensive work in the area of VLSI physical design. He developed a suite of distributed place-and-route tools for VLSI and field-programmable gate-array (FPGA) chips. For six years, he has been an Integrated Circuit Designer for semiconductor companies. He currently leads ultrafast circuit design activities for nano and quantum electronic devices. He has successfully developed computer-aided design (CAD) tools for high-performance VLSI circuit simulation (NDR SPICE) and numerous circuit topologies for quantum MOS and other quantum-well devices. Several U.S. and Japanese semiconductor companies, including Texas Instruments Incorporated, Hughes Research Laboratory, Lockheed-Martin, NTT, and NEC, have collaborated with him on this research. He was guest editor of the June 1994 "Special Issue On Memory Testing And Reliability" of Journal of Electronic Testing-Theory and Applications.

Dr. Mazumder is a member of Sigma Xi, Phi Kappa Phi, and the Association for Computing Machinery (ACM) Special Interest Group on Design Automation (SIGDA). He was guest editor of the March 1993 "Special Issue on Multimegabit Memory Testing" of the IEEE Design and Test of Computers. He was the guest editor of two special issues on "Emerging Nanoelectronic Technologies and Their Applications" of the IEEE TRANSACTIONS ON VERY LARGE SCALE INTEGRATION SYSTEMS (December 1997) and the PROCEEDINGS OF THE IEEE (April 1998). He is on the Editorial Board of the PROCEEDINGS OF THE IEEE and is an associate editor of the IEEE TRANSACTIONS ON VERY LARGE SCALE INTEGRATION SYSTEMS. He was a recipient of Digital's Incentives for Excellence Award, the BF Goodrich National Collegiate Invention Award, the National Science Foundation Research Initiation Award, and Bell Northern Research Laboratory Faculty Award. 